\title{
Développer nos relations scientifiques avec les pays d'Extrême-Orient : un impératif
}

Au cours de sa longue existence, la Société Française de Physique a été amenée à tisser de nombreux liens, plus ou moins proches, avec les sociétés sœurs des pays voisins, d'abord dans un cadre bilatéral, mais aussi, depuis 1968, dans un cadre multilatéral au sein de la Société Européenne de Physique (EPS). Elle en a également avec les sociétés savantes américaines, mais jusqu'à présent peu de relations ont été établies avec les sociétés scientifiques du continent asiatique. Dans un précédent éditorial [1], j'avais évoqué l'émergence exponentielle du potentiel scientifique des nations asiatiques, de façon concomitante avec le développement de leur puissance économique. Ces derniers mois, à l'occasion de différents séjours en Extrême-Orient (Chine, Japon, Singapour...), j'ai pu constater les changements s'opérant continûment et de façon extrêmement rapide dans les capacités de recherche de ces pays. Selon un récent rapport de I'UNESCO, la Chine a ainsi pris la deuxième place dans la production scientifique mondiale (en termes de publications et de formation supérieure) derrière les USA, qui voient peu à peu les pays asiatiques grignoter leur traditionnelle suprématie.

En Europe, I'EPS en particulier a pris conscience de la nécessité d'intensifier et de diversifier ses relations avec ces nations, et d'y augmenter sa présence. À la suite d'une première rencontre organisée à Tsukuba (Japon) en 2010, un second sommet Europe-Asie (ASEPS, Asia Europe Physics Summit) a eu lieu à Wroclaw (Pologne) en octobre 2011, sous l'égide de l'EPS et de I'AAPPS (Association of Asia Pacific Physical Societies) - la SFP étant coorganisatrice. Les débats ont été très ouverts, abordant les divers aspects de la coopération scientifique ainsi que les problèmes sociétaux. Cinq ateliers de travail s'y sont tenus : ils couvraient la coopération en physique des grandes infrastructures de recherche, l'organisation en réseau, les défis scientifiques et socio-économiques (énergie, environnement, santé...), l'éducation et la formation scientifiques (écoles et ateliers de travail communs), la mobilité réciproque et un programme d'échange équilibré pour étudiants et jeunes chercheurs, les liens entre recherches académique et industrielle. Le côté financier n'a été que peu abordé et des ressources devront être trouvées auprès des différentes agences de moyens et organismes de recherche nationaux et internationaux (Commission européenne, etc.) Une déclaration finale, sous forme de mémorandum, a été signée par l'EPS et l'AAPPS [2]. Le prochain sommet est prévu au printemps 2013, et devrait être préparé lors de réunions plus spécialisées sur des aspects particuliers de la coopération Europe-Asie.

En parallèle, la SFP a décidé de développer et intensifier ses relations bilatérales avec les sociétés savantes partenaires en Asie. Des conventions de coopération sont en cours de discussion avec les sociétés japonaises de physique ( 18000 membres) et de physique appliquée ( 25000 membres), que j'ai rencontrées début novembre au Japon, ainsi qu'avec l'Institut de Physique de Singapour (IPS). Un atelier de travail commun IPS-SFP se tiendra à Singapour les 16-19 janvier 2012 [3]. Centré sur l'information quantique ("Condensed-matter and quantum information physics: shedding new light with atomic systems"), il réunira sept orateurs du côté français et autant du côté singapourien. Cet atelier, organisé en partie par les divisions PAMO et Matière condensée de la SFP, est patronné et soutenu par I'Ambassade de France, le Triangle de la Physique et l'École polytechnique, ainsi que par les deux universités de Singapour. Ces opérations de coopération scientifique avec les sociétés asiatiques de physique devraient continuer de se diversifier dans le futur. Avec l'extension de ses collaborations vers l'Extrême-Orient, la Société Française de Physique peut espérer gagner en termes de visibilité internationale, profiter aussi de l'expérience de sociétés savantes où l'adhésion individuelle des scientifiques s'opère naturellement, et maintenir un contact étroit avec des pays en émergence rapide.

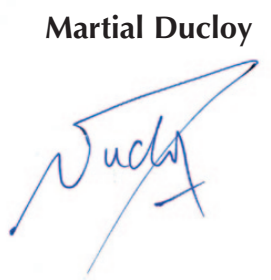

[1] Reflets de la physique n²0 (juillet-août 2010), p. 2.

[2] www.eps.org/news/asia-europe-physics-summit-wroclaw-pl

[3] www.ntu.edu.sg/ias/upcomingevents/IPS2012/Pages/HomePage.aspx 